\title{
Systems engineering in context
}

\author{
Peter A. Beling ${ }^{1} \cdot$ Cody H. Fleming ${ }^{1} \cdot$ William T. Scherer $^{1}$
}

Published online: 4 June 2019

(c) Springer Science+Business Media, LLC, part of Springer Nature 2019

This issue of Environment Systems \& Decisions features papers developed from the 16th Annual Conference on Systems Engineering Research (CSER 2018), held May 8-9, 2018 at the University of Virginia, Charlottesville, Virginia. CSER 2018 offered researchers in academia, industry, and government a common forum to present, discuss, and influence systems engineering research. The conference provided access to forward-looking research by renowned academicians as well as perspectives from senior industry and government representatives. Co-founded by the University of Southern California and Stevens Institute of Technology in 2003, the CSER series of conferences has become the preeminent event for researchers in systems engineering across the globe.

The conference theme of Systems Engineering in Context was intended to highlight the ways that context-economic, social, cultural, organizational, and otherwise-can shape decision problems and other aspects of systems engineering. In their introduction to the inaugural issue of Environment Systems and Decisions, Linkov, Lambert, and Collier (2013) stated the first word in the title of the journal "is intended to imply not only the natural environment, but also the environment in which a problem, decision, or innovation exists." The focus of CSER 2018 on the context of decision-making and systems engineering is in keeping with this usage of the word environment.

Topics for CSER 2018 included:

- Systems in context:

Peter A. Beling

pb3a@virginia.edu

Cody H. Fleming

fleming@virginia.edu

William T. Scherer

wts@ virginia.edu

1 Department of Engineering Systems and Environment, University of Virginia, 151 Engineer's Way, P.O. 70047, Charlottesville, USA
- Formative methods: requirements

- Integration, deployment, assurance

- Human factors

- Safety and security

- Decisions/control and design; systems modeling:

- Optimization, multiple objectives, synthesis

- Risk and resiliency

- Collaborative autonomy

- Coordination and distributed decision-making

- Prediction:

- Prescriptive modeling; state estimation

- Stochastic approximation, stochastic optimization, and control

- Integrative data engineering:

- Sensor management

- Design of experiments

The papers in this issue reflect on the theme of Systems Engineering in Context, developing and implementing methods from set-based design, recommending principles of model validation, and characterizing the complex relationships between engineered technology and people.

Buchanan et al. (2019) describe the traditional notions of point-based design, where the systems engineering process aims to quickly converge to a single solution. They argue that this approach is brittle with respect to the time, cost, and optimality of the systems engineering process. They then advocate for set-based design, which for consideration of all solutions within given parameters enables filtering of possibilities to converge at a final solution that may be overlooked by point-based design. After developing and integrating a cost model with an engineering model, the authors demonstrate the approach of set-based design on a light reconnaissance vehicle. 
Wade et al. (2019) provide a different, yet complementary, view of set-based design to those of Buchanan et al. (2019). The authors motivate this topic by describing the ability to eliminate less promising sets of designs and identifying more promising design sets using formal mathematical techniques. They then describe the use of set-based design in the context of system resilience and develop a technique called Convergent Set-based Design. The authors describe a procedure for mathematical elimination and improvement of sets of designs, as well as the ability to measure and quantify resilience of systems using probability trees. They then describe the use of dominance identification in set-based design that compares statistical means for value and cost.

Stenholm et al. (2019) present a framework for technology reuse. First, the authors argue that the reuse of technology constitutes an important organizational capability and can result in a competitive advantage, particularly in domains where market demand constantly evolves. However, there exist many practices that support technology reuse, and a general assessment of these practices has been lacking. Therefore, the authors review and analyze technology reuse across the four dimensions of strategy, process, culture, and information technology. The framework provides a theoretical overview across these domains and is illustrated using several real-world use cases.

Collier and Lambert (2019) motivate the importance of model validation in systems engineering, and then advocate for the systems engineering discipline as a major stakeholder in advancing the theory and practice of model validation. After defining model validation and its principles, they then define the current state of the art and challenges in validating models, particularly those of complex systems. They conclude that the validity of a model can be determined as a function of the amount of resources spent in constructing the model as well as its ability to reduce the amount of risk exposure for the model's user(s).
Finally, Vacca (2019) argues for the importance of understanding the social context in which technological systems operate that goes beyond rational choice models of human behavior. Motivating this important topic with historical examples of the Royal Navy, he provides a review of how humans are typically modeled within systems theory and formal systems engineering. He then describes both the introduction of new technology into complex systems-ofsystems, as well as the changing nature of human roles as these technologies come online. Next, Vacca describes the context of these technologies' operational social systems, and their failures to keep pace with technological change. He not only concludes with an argument about the potential for systems theory to account for these failures but also advocates for a paradigm shift away from rational models of operational decision-making.

\section{References}

Buchanan RK, Richards JE, Rinaudo CH et al (2019) Integrating setbased design into cost analysis. Environ Syst Decis. https://doi. org/10.1007/s10669-019-09729-z

Collier ZA, Lambert JH (2019) Principles and methods of model validation for model risk reduction. Environ Syst Decis. https://doi. org/10.1007/s10669-019-09723-5

Linkov I, Lambert JH, Collier ZA (2013) Introduction to the inaugural issue of environment systems and decisions. Environ Syst Decis 33(1):1-2. https://doi.org/10.1007/s10669-013-9434-9

Stenholm D, Stig DC, Ivansen L, Bergsjö D (2019) A framework of practices supporting the reuse of technological knowledge. Environ Syst Decis. https://doi.org/10.1007/s10669-019-09732-4

Vacca WA (2019) The social context of technological systems: dreadnoughts, computers, and flags. Environ Syst Decis. https://doi. org/10.1007/s10669-019-09722-6

Wade Z, Parnell G, Goerger S, Pohl E, Specking E (2019) Convergent set-based design for design of complex resilient systems. Environ Syst Decis. https://doi.org/10.1007/s10669-019-09731-5 